\title{
Association of cigarette smoking, obesity, and underlying medical conditions with COVID-19 hospitalization and mortality in Iran: A nationwide retrospective ecological study
}

\author{
Zahra Khorrami ${ }^{1}$, Sairan Nili ${ }^{2}$, Hamid Sharifi ${ }^{1}$, Sana Eybpoosh*3 (1), Mostafa Shokoohi ${ }^{1,4}$ \\ Received: 11 Jun 2020 \\ Published: 8 Oct 2020
}

Abstract

Background: Coronavirus Disease 2019 (COVID-19) has resulted in a considerable number of deaths worldwide. This ecological study aimed to explore the relationship between COVID-19 hospitalization and mortality with smoking, obesity, and underlying conditions in Iran.

Methods: Provincial-level COVID-19 data were obtained from the official reports. Two outcomes were assessed: the total number of hospitalizations and deaths. Data on underlying health conditions, cigarette smoking, and obesity were obtained from national surveys. Negative binomial regression was used to report incident rate (IRR) ratios.

Results: As of April 22, 2020, a total number of 43950 lab-confirmed COVID-19 hospitalizations and 5391confirmed COVID-19 deaths were officially reported. Adjusting for underdetection to cover the number of clinically-confirmed COVID-19 cases, a total of 76962 additional hospitalizations (ie, total lab- and clinically-confirmed hospitalizations $=120912 ; 175 \%$ increase) and 7558 additional deaths (ie, total lab- and clinically-confirmed deaths $=12949 ; 140 \%$ increase) were estimated during the same period. Provinces with a higher prevalence of obesity (IRR: $2.75,95 \%$ CI: $1.49,5.10)$, cigarette smoking $(1.81 ; 95 \%$ CI: 1.01, 3.27), hypertension (1.88; 95\% CI: 1.03, 3.44), and diabetes mellitus $(1.74 ; 95 \%$ CI: $0.96,3.16)$ had a higher likelihood of COVID-19 death rates.

Conclusion: Inequality in COVID-19 hospitalization and mortality was observed in provinces whose populations had underlying diseases, in particular, obesity, cigarette smoking, hypertension, and diabetes.

Keywords: Underlying conditions, COVID-19, Hospitalization, Mortality, Ecological study, Iran

Conflicts of Interest: None declared

Funding: None

\section{*This work has been published under CC BY-NC-SA 1.0 license.}

Copyright $\subseteq$ Iran University of Medical Sciences

Cite this article as: Khorrami Z, Nili S, Sharifi H, Eybpoosh S, Shokoohi M. Association of cigarette smoking, obesity, and underlying medical conditions with COVID-19 hospitalization and mortality in Iran: A nationwide retrospective ecological study. Med J Islam Repub Iran. 2020 (8 Oct);34:133. https://doi.org/10.47176/mjiri.34.133

\section{Introduction}

The Coronavirus Disease 2019 (COVID-19) was first emerged in Wuhan, China, in late 2019 and has rapidly
Corresponding author: Dr Sana Eybpoosh, s_eybpoosh@pasteur.ac.ir

1. HIV/STI Surveillance Research Center, and WHO Collaborating Center for HIV Surveillance, Institute for Futures Studies in Health, Kerman University of Medical Sciences, Kerman, Iran

2. Department of Biostatistics and Epidemiology, School of Public Health, Kerman University of Medical Sciences, Kerman, Iran

3. Department of Epidemiology and Biostatistics, Research Centre for Emerging

and Reemerging Infectious Diseases, Pasteur Institute of Iran, Tehran, Iran

4. Dalla Lana School of Public Health, University of Toronto, Toronto, Canada $\uparrow$ What is "already known" in this topic:

Coronavirus Disease 2019 (COVID-19) has resulted in a considerable number of deaths worldwide. Factors such as older age ( $>65$ years old), being male, and having comorbidity and underlying medical conditions could put infected individuals at greater risk of death.

\section{$\rightarrow$ What this article adds:}

This ecological study explored the relationship between COVID-19 hospitalization and mortality with smoking, obesity, and underlying conditions in Iran. Provinces with a higher prevalence of obesity (IRR: 2.75), cigarette smoking (1.81), hypertension (1.88), and diabetes mellitus (1.74) had a higher likelihood of COVID-19 death rates. Inequality in COVID-19 hospitalization and mortality was observed in provinces whose populations had underlying diseases. 
spread to other parts of the world $(1,2)$. On January 30 , 2020, WHO identified COVID-19 as an international public health emergency and declared it as a pandemic (3). In Iran, the first confirmed cases were officially reported in February 20, 2020. The disease has circulated quickly throughout the country, such that by April 20, 2020, more than 8000 confirmed infected cases and 5209 confirmed deaths were reported. However, it is expected that both the total number of infected and deaths could be higher than the official reports, as the clinical symptoms of the disease are not specific and a large portion of the infections are asymptotic. Moreover, the capacity of the country to test and identify the whole cases has not been enough, particularly at the beginning of the epidemic (4).

The global cases fatality rate of COVID-19 is reported around $2 \%$, with substantial variations across different subpopulations (5). Factors such as older age ( $>65$ years old), being male, and having comorbidity and underlying medical conditions (eg, diabetes, hypertension, chronic obstructed pulmonary disease (COPD), and obesity) could put infected individuals at greater risk of death (6). Geographical variations in case fatality have also been reported (7), such that some countries could have a higher fatality rates relative to some other countries. The evidence indicates that a part of the variabilities in case fatality rates could be due to the higher extent of the comorbidities; for example, the higher fatality rate of the infection in Italy compared to China could be associated with the higher prevalence of the obesity among adults in Italy (8).

One of the main important issues of emerging and reemerging outbreaks and pandemic is to explore what risk factors or determinants could be associated with their burden, such as the total number of cases, hospitalization, and deaths (9). Identifying these associated factors could help better manage the epidemics and reduce their burden. However, determining and identifying these factors could be difficult due to the lack of individual-level data. On the other hand, ecological studies that rely on group-level data could guide policymakers and researchers to generate hypotheses to evaluate the impact of these determinants (10). Unlike the individual-level data used for the identification of risk factors in other observational studies (eg, longitudinal studies), ecological studies aim to obtain such associations using the comparison of aggregate data. In this study we aimed to explore the association of some underlying conditions and health risk behaviors/factors with 2 main COVID-19-related outcomes (hospitalization and deaths) in Iran using province-level data. To the best our knowledge, no study has been conducted with an ecological design to address the risk determinants of the COVID19 outcomes in Iran.

\section{Methods}

\section{Study population and area}

The study was conducted across all 31 provinces of Iran from the official onset of the COVID-19 epidemic (Feb 19, 2020) through April 22, 2020. Due to the ecological nature of the study, the units of the analyses were provinces. Thus, we used province-level data for 2 COVID-19associated outcomes (eg, hospitalization and death rates) and the underlying medical conditions and risk factors.

\section{COVID-19-associated outcomes}

Two outcomes were examined: (a) The COVID-19associated hospitalizations, defined as the total number of individuals hospitalized due directly to COVID-19 divided by the total population, presented per 100000 in each province, and (b) COVID-19 deaths, defined as the total number of confirmed and probable deaths due to COVID19 divided by the total population, presented per 100000 in each province. Reliance only on confirmed cases might be prone to underestimation of these 2 outcomes. Thus, we took into account 2 types of hospitalized and death cases: (i) only confirmed cases: patients whose polymerase chain reaction (PCR) test results were positive for COVID-19; (ii) probable cases, who were those patients assumed to be clinically-confirmed COVID-19 cases. Given low sensitivity of laboratory tests, the number of probable (clinically-confirmed) COVID-19 hospitalizations and deaths was also considered in the analyses. Data for the lab-confirmed cases were obtained from the national COVID-19 reports from the Ministry of Health. The second type of cases (ie, probable cases) were assumed to be those patients who were diagnosed by the clinicians, but not using a PCR. Clinically-confirmed COVID-19 cases were diagnosed using the examination results of the lung imaging features of the chest computed tomography (CT) scan showing ground glass pathognomonic features consistent with Coronavirus pneumonia (11), or based on the clinical judgment of the clinician about patient's symptoms. Finally, we added the additional probable cases to the total number of confirmed cases and used them as the final variable utilized for the statistical analyses.

Morbidity or underlying medical conditions data

Except for cancers, province-level aggregate underlying medical conditions data were obtained from the seventh large-scale cross sectional surveys according to the 2016 STEPwise approach in surveillance of noncommunicable diseases risk factor. The study protocol and sampling methods are described elsewhere (12). In brief, STEPS is a simple, standardized approach for collecting, analyzing, and disseminating data in WHO member countries. Also, it has been designed to provide nationally and provincially representative estimates on several health risk factors and noncommunicable diseases of Iranians aged 18 years and older recruited from both urban and rural areas. Participants were sampled through using stratified random cluster sampling, proportionate to the population size of each province. Overall, 31050 individuals were recruited, of them $30541(98.4 \%)$ completed the first step (the questionnaire-based assessment) and 30042 (96.7\%) completed the second step (the physical measurement). For the third step of the survey (the biochemical measurement), only individuals aged 25 years and older were invited (89.3\%), of whom 19778 took part in this step (response rate: $71.3 \%$ ). Weights to account for nonresponse bias were incorporated into the survey analysis. Data on having any types of cancers were obtained from the National Cancer Registration Program in 2015 (13). 


\section{Smoking and body mass index}

Cigarette smoking was defined as ever daily cigarette use. We used body mass index (BMI) to measure obesity. BMI was calculated by dividing participants' weight $(\mathrm{kg})$ to the square of height $\left(\mathrm{m}^{2}\right)$. Obesity was defined as BMI $\geq 30 \mathrm{~kg} / \mathrm{m}^{2}$. Measurements were implemented by standard operating procedures and performed by trained personnel (14).

\section{Underlying medical conditions}

Diabetes mellitus type II (DMT2) was defined as having an $\mathrm{HbA} 1 \mathrm{c} \geq 6.4 \%$, or self-reported oral use of hypoglycemic agents, or taking insulin. Hypertension was defined as systolic blood pressure $\geq 140 \mathrm{mmHg}$ or diastolic blood pressure $\geq 90 \mathrm{mmHg}$ in the mean of 2 consecutive measurements or self-reported use of antihypertensive medications. Having any cardiovascular diseases was defined as self-reported history of heart attack and/or stroke as 2 major cardiovascular diseases. Having asthma and/or wheezing were recorded based on conditions. Finally, the agespecific rate (ASR, per 100000 populations) of any type of cancers in both genders was obtained from the latest report.

\section{Statistical analysis}

Each province was treated as a single observation. The 2 main study outcomes, the COVID-19-associated hospitalization rates and death rates, were mapped to show the geographical variations across the study provinces. Then, as per ecological studies, a correlation analysis using the Pearson's correlation test was performed between the 2 study outcomes and the underlying medical conditions and the 2 morbidities. To do this, the variables were dealt as continuous. Also, scatter plots were generated to visualize the pattern of these correlations. Second, given the nature of the study outcomes (both were count data), the negative binomial (NB) regression models were performed to estimate the incidence rate ratio (IRRs) and their 95\% confidence intervals (CI). Negative binomial models were deemed appropriate because of the overdispersion of data for both COVID-19-associated hospitalization and death. For ease of interpretations, all the explanatory variables were treated as dichotomous: below the median (code 0 ) an above the median (code 1). We reported IRRs for the provinces above the median versus those below the median. In these models, the total population of each province was considered the offset. Statistical analyses were performed in Stata v 14.0 (Stata crop, Texas, USA). P value less than 0.05 was considered significant, while $\mathrm{P}$ values between 0.05 and 0.10 were considered as borderline significant.

\section{Ethical considerations}

Ethical approval for this research was obtained from the Research Ethic Committee, Kerman University of Medical Sciences (Code: IR.KMU.REC.1398.735). No written or oral consent form was needed to be obtained from the patients, as this study and its data relied on officially reported administrative data.

\section{Results}

As of April 22, 2020, a total of 43950 lab-confirmed COVID-19 cases were reported in all the 31 provinces of Iran, with provincial-level incidence rates ranging the lowest at Sistan-and-Baluchistan province, located in the southeast of Iran, and the highest in the Golestan province, located in the northeast of the country. We estimated a total of 76962 additional COVID-19 hospitalizations, resulting in a total of 120912 confirmed and probable hospitalizations together ( $\sim 175 \%$ increase).

In addition, 5391 lab-confirmed COVID-19 deaths were officially reported within the same period. Provincial-level rates were lowest in Boushehr province in the south of the country and highest in Qom province in the center. We assumed that 7558 additional deaths might have occurred due to COVID-19, considering those whose positive tests were diagnosed by the clinicians, but they did not have a PCR test result. The addition of lab-confirmed cases and clinically-confirmed cased resulted in a total of 12949 deaths (ie, $\sim 140 \%$ increase).

\section{Correlations and associations with COVID-19 hospital- izations}

COVID-19 hospitalization had only a borderline significant correlation with incidence of all cancers $(\mathrm{r}=0.35$, $\mathrm{p}=0.057$ ). The correlation of other explanatory variables with hospitalization was not statistically significant, even though obesity $(\mathrm{r}=0.13, \mathrm{p}=0.475)$, diabetes $(\mathrm{r}=0.11$, $\mathrm{p}=0.588)$, and asthma and wheezing $(\mathrm{r}=0.05, \mathrm{p}=0.8 .06)$ were positively correlated and smoking $(\mathrm{r}=-0.07$, $\mathrm{p}=0.718)$ and cardiovascular diseases $(\mathrm{r}=0-0.05, \mathrm{p}=0.786)$ were negatively correlated (Table 1, Fig. 1).

Table 1. Pearson correlation of the underlying medical conditions associated with COVID-19 hospitalization and deaths in Iran, 2020

\begin{tabular}{|c|c|c|c|c|}
\hline \multirow[b]{3}{*}{ Underlying medical conditions and risk factors } & \multicolumn{2}{|c|}{ COVID-19-associated Hospitalization } & \multicolumn{2}{|c|}{ COVID-19-associated Death } \\
\hline & Correlation & $\mathrm{p}$ & Correlation & $\mathrm{p}$ \\
\hline & & & & \\
\hline Obesity & 0.13 & 0.475 & 0.26 & 0.156 \\
\hline Smoking & -0.07 & 0.718 & 0.11 & 0.553 \\
\hline Diabetes (HbA1C) & 0.11 & 0.588 & 0.15 & 0.433 \\
\hline Hypertension & 0.00 & 0.999 & 0.06 & 0.734 \\
\hline Asthma and Wheezing & 0.05 & 0.806 & 0.31 & 0.092 \\
\hline Cardiovascular diseases & -0.05 & 0.786 & -0.14 & 0.446 \\
\hline Cancers (any) & $0.35^{\mathrm{a}}$ & 0.057 & 0.15 & 0.433 \\
\hline
\end{tabular}


A

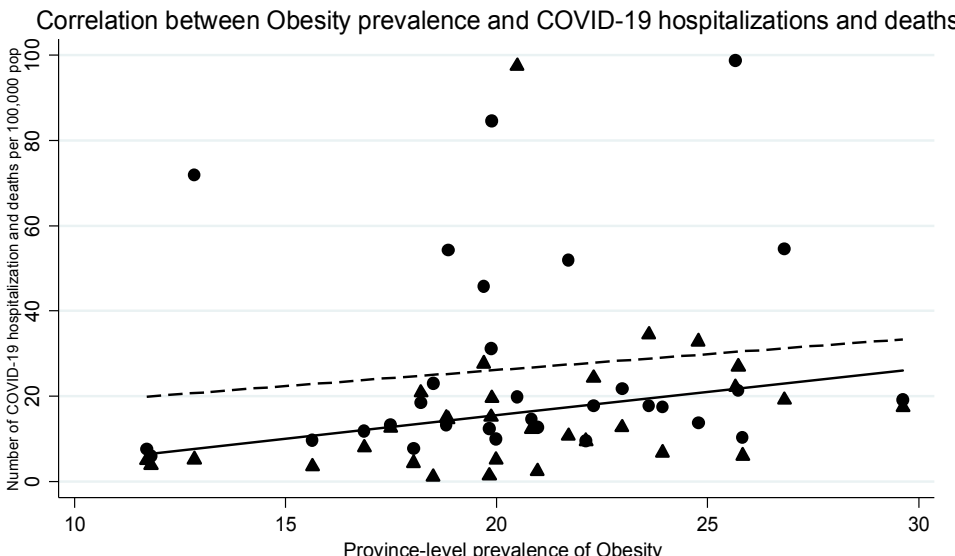

- COVID-19 hospitalizations $\Delta \quad$ COVID-19 deaths - - - - fitted line for COVID-19 hospitalizations — fitted line for COVID-19 deaths R-squared for CoVID-19 hospitalizations $=0.0177$, R-squared for COVID-19 Deaths $=0.0682$

$\mathrm{C}$

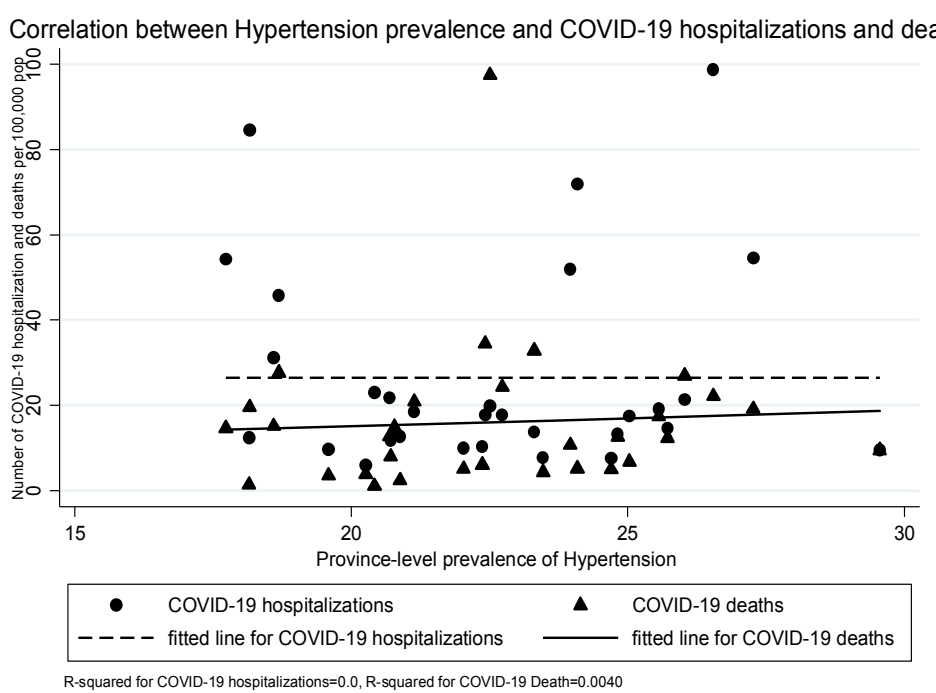

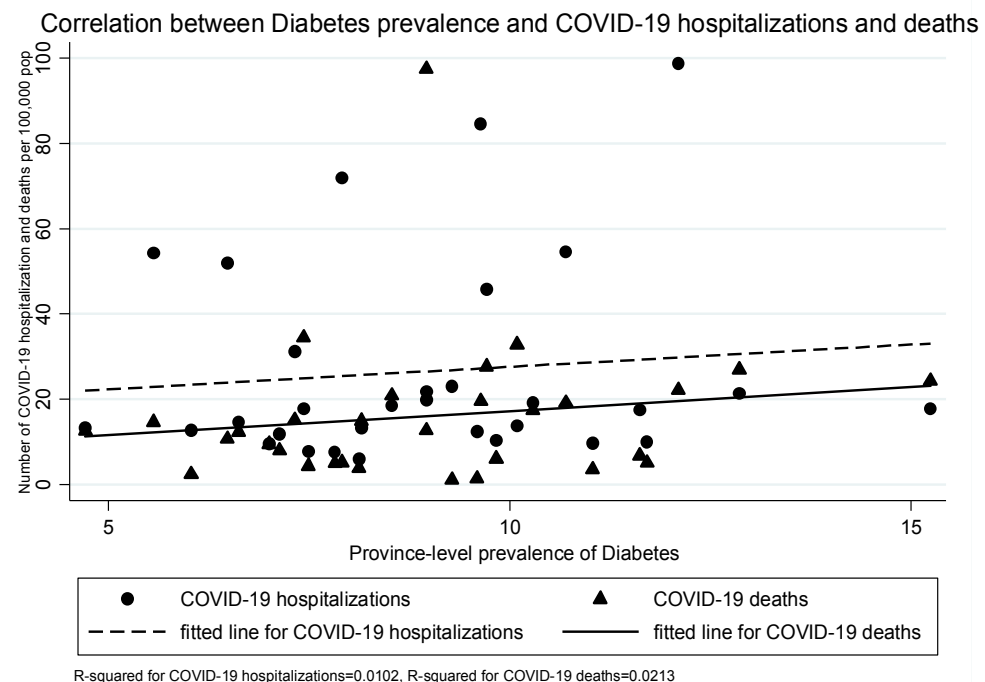

D

Correlation between Smoking prevalence and COVID-19 hospitalizations and deaths

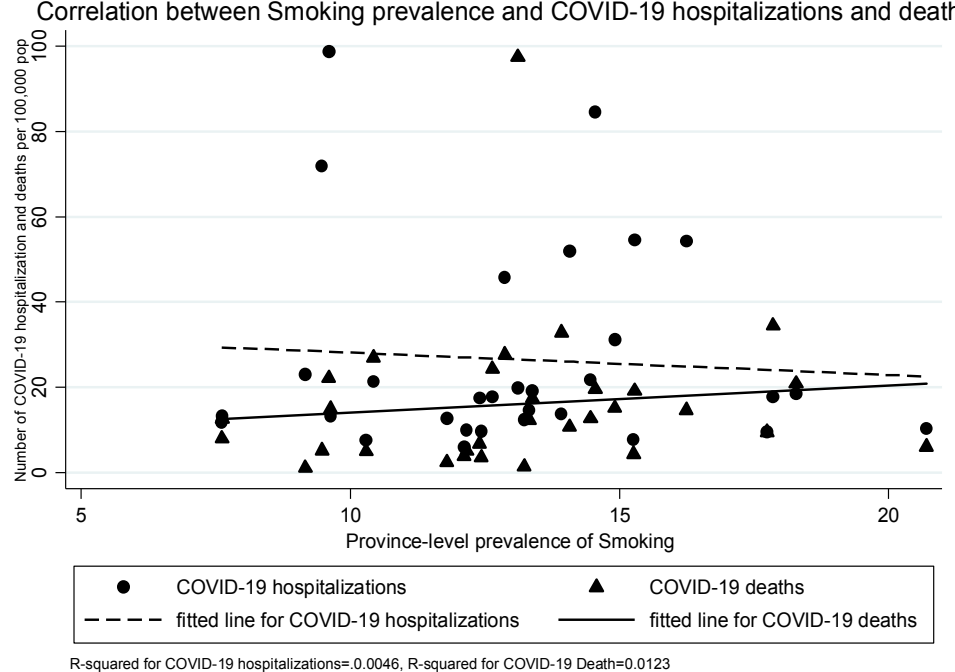




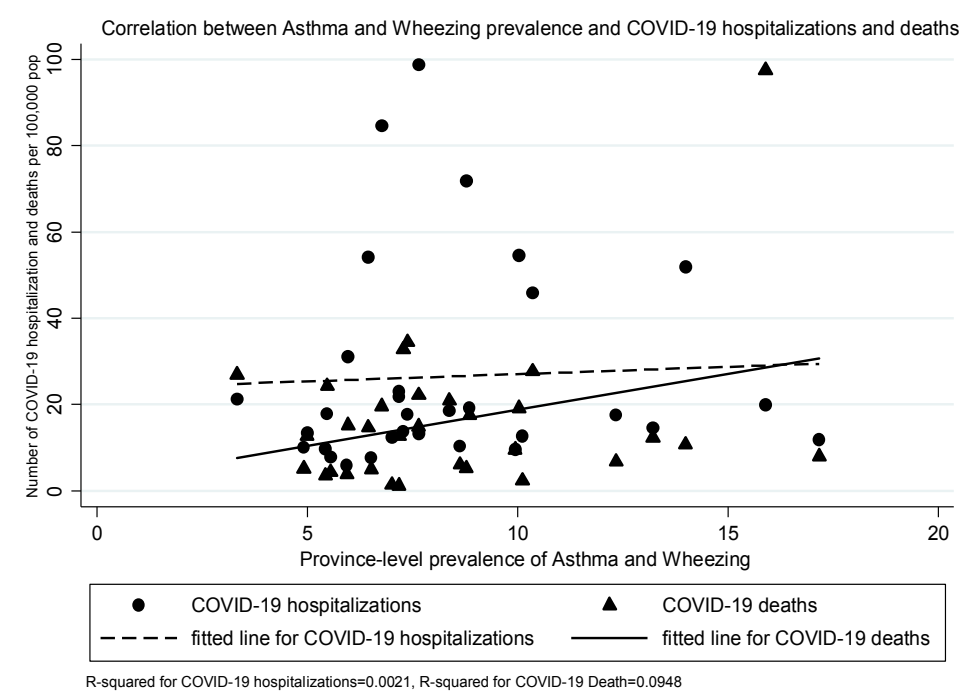

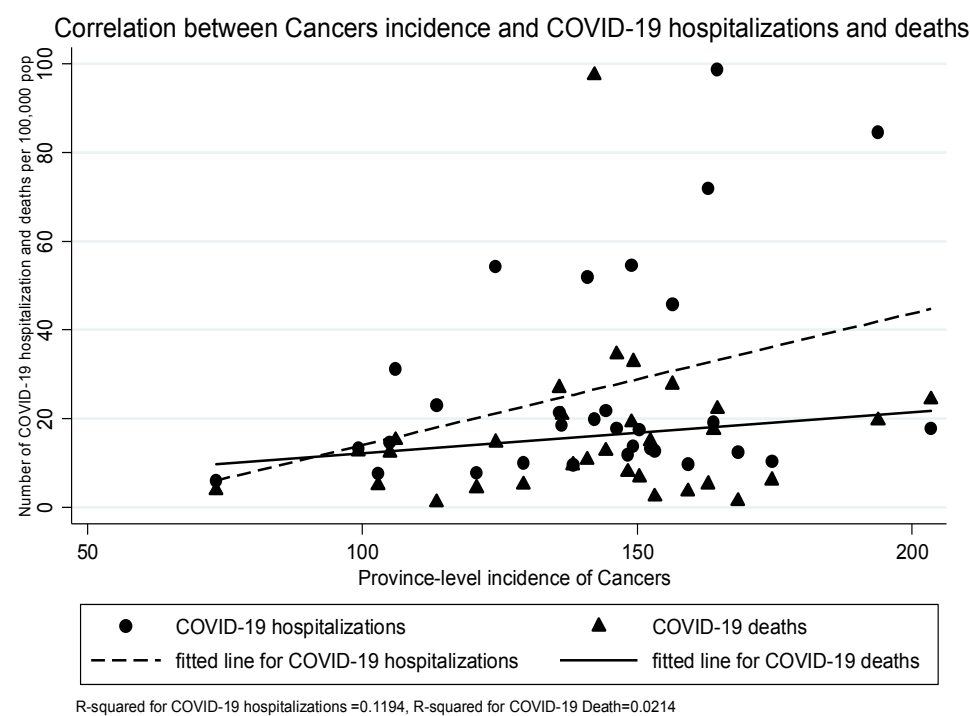

Fig. 1. The provincial-level correlation of COVID-19 hospitalization and death rates with the prevalence of obesity (A), diabetes mellitus type 2 (B), hypertension (C), smoking (D), asthma and wheezing (E) and cancer (F) (Iran, 2020). 
Table 2. The negative binomial regression analyses of the underlying medical conditions associated with COVID-19 deaths in Iran, 2020

\begin{tabular}{|c|c|c|c|c|}
\hline & \multicolumn{2}{|c|}{ COVID-19 Hospitalization } & \multicolumn{2}{|c|}{ COVID-19 Death } \\
\hline & $\begin{array}{c}\text { IRR } \\
(95 \% \mathrm{CI})^{\mathrm{a}} \\
\end{array}$ & $\mathrm{p}$ & $\begin{array}{c}\text { IRR } \\
(95 \% \mathrm{CI}) \\
\end{array}$ & $\mathrm{p}$ \\
\hline Obesity & & & & \\
\hline Below median (from $11.7 \%$ to $19 \%$ ) & Ref & & Ref & \\
\hline Above median (from $19.1 \%$ to $29.6 \%$ ) & $\begin{array}{c}1.58 \\
(0.91,2.77)\end{array}$ & 0.110 & $\begin{array}{c}2.75 \\
(1.49,5.10)^{\mathrm{c}}\end{array}$ & 0.001 \\
\hline Smoking & & & & \\
\hline Below median (from $7.6 \%$ to $13 \%$ ) & Ref & & Ref & \\
\hline Above median (from $13.1 \%$ to $20.7 \%$ ) & $\begin{array}{c}1.08 \\
(0.65,1.84)\end{array}$ & 0.748 & $\begin{array}{c}1.81 \\
(1.01,3.27)\end{array}$ & 0.047 \\
\hline Hypertension & & & & \\
\hline Below median (from $17.7 \%$ to $22.4 \%$ ) & Ref & & Ref & \\
\hline Above median (from $22.5 \%$ to $29.5 \%$ ) & $\begin{array}{c}0.95 \\
(0.56,1.64)\end{array}$ & 0.876 & $\begin{array}{c}1.88 \\
(1.03,3.44)\end{array}$ & 0.039 \\
\hline Diabetes (HbA1C) & & & & \\
\hline Below median (from $4.7 \%$ to $8.8 \%$ ) & Ref & & Ref & \\
\hline Above median (from $8.9 \%$ to $15.3 \%$ ) & $\begin{array}{c}1.31 \\
(0.78,2.21)\end{array}$ & 0.295 & $\begin{array}{c}1.74 \\
(0.96,3.16)\end{array}$ & 0.066 \\
\hline Asthma and Wheezing & & & & \\
\hline Below median (from $3.3 \%$ to $7.4 \%$ ) & Ref & & Ref & \\
\hline Above median (from $7.5 \%$ to $17.5 \%$ ) & $\begin{array}{c}1.16 \\
(0.67,2.01)\end{array}$ & 0.580 & $\begin{array}{c}1.48 \\
(0.78,2.79)\end{array}$ & 0.224 \\
\hline Cancers (any) & & & & \\
\hline Below median (from 73.4 to 146 ) & Ref & & Ref & \\
\hline Above median (from 146.1 to 203.5 ) & $\begin{array}{c}1.54 \\
(0.93,2.56)\end{array}$ & 0.092 & $\begin{array}{c}0.91 \\
(0.49,1.69)\end{array}$ & 0.774 \\
\hline Cardiovascular diseases & & & & \\
\hline Below median (from $3.7 \%$ to $8.5 \%$ ) & Ref & & Ref & \\
\hline Above median (from $8.6 \%$ to $13.5 \%$ ) & $\begin{array}{c}1.60 \\
(0.97,2.65)\end{array}$ & 0.066 & $\begin{array}{c}1.03 \\
(0.55,1.91)\end{array}$ & 0.918 \\
\hline
\end{tabular}

The results of the regression analyses showed that provinces whose populations had higher prevalence of the underlying conditions had a higher likelihood of hospitalization. Specifically, provinces with higher incidence of cancers $(\mathrm{IRR}=1.54,95 \% \mathrm{CI}: 0.93,2.56 ; \mathrm{p}=0.092)$ and prevalence of cardiovascular diseases $(1.60,95 \% \mathrm{CI}$ : $0.97,2.65$; $\mathrm{p}=0.066$ ) had higher likelihood of COVID-19-associated hospitalization rates, even though both were borderline significant. Provinces with higher prevalence of other explanatory variables, including obesity, smoking, hypertension, diabetes, and asthma and wheezing are presented in Table 2.

\section{Correlations and associations with COVID-19 deaths}

We found that provinces with higher asthma and wheezing prevalence were positively correlated with increases in COVID-19 death rates, with a borderline significance $(r=0.31, p=0.092)$. The correlation between other explanatory variables with death rates was not statistically significant, with obesity $(\mathrm{r}=0.26, \mathrm{p}=0.156)$, smoking $(\mathrm{r}=0.11$, $\mathrm{p}=0.553)$, and diabetes $(\mathrm{r}=0.15, \mathrm{p}=0.443)$, hypertension $(\mathrm{r}=0.06, \mathrm{p}=0.734)$, and cancers $(\mathrm{r}=0.15, \mathrm{p}=0.433)$ being positively correlated, and cardiovascular diseases $(\mathrm{r}=-0.14$, $\mathrm{p}=0.446$ ) being negatively correlated (Table 1, Fig. 1).

Regression analyses showed that provinces with higher prevalence of the underlying conditions had a higher likelihood of hospitalization. Specifically, provinces with higher prevalence of obesity (IRR $=2.75,95 \% \mathrm{CI}$ : 1.49 , 5.10; $\mathrm{p}=0.001)$, smoking $(1.81,95 \% \mathrm{CI}: 1.01,3.27$; $\mathrm{p}=0.047)$, and hypertension $(1.88,95 \% \mathrm{CI}: 1.03,3.44$; $\mathrm{p}=0.039$ ) were significantly associated with higher death rates. Provinces with higher rates of other explanatory variables were not significantly associated with higher or lower rates of COVID-19 death (Table 2).

\section{Discussion}

This ecological analysis in Iran documented that the provinces with higher underlying medical conditions had a higher likelihood of COVID-19 burden. Moreover, greater prevalence of obesity, cigarette smoking, hypertension, and diabetes mellitus were significantly associated a higher likelihood of COVID-19 death rates. Weak statistical associations were found between hospitalization rates and underlying condition, particularly, with cancers and cardiovascular diseases. While the same pattern of association was found when we used data only for lab-confirmed cases (data not shown but available upon request) and clinically-confirmed cases, the way we presented and corrected the COVID-19 data could demonstrate a better picture of the actual burden of this disease in Iran.

In line with the existing literature indicating the association of obesity and smoking with COVID-19 burdens, we also found a significant association of these two factors with COVD-19-associated mortality. Inequality in COVID-19 mortality in older adults in Italy compared with China was explained by the higher prevalence of obesity among Italians (8). Severe obesity, in addition to causing a number of chronic diseases, increases the risk of severe forms of COVID-19 and often causes serious breathing problem called acute respiratory distress syndrome (ARDS) (15), as well as reduces expiratory reserve volume and functional capacity (8). The growing body of 
evidence has also highlighted the negative association of smoking with COVID-19 outcomes. A recent systematic review showed that smoking is presumably related to the negative progression and adverse outcomes of COVID-19, such that smokers relative to nonsmokers were 1.4 times more likely to have severe symptoms of COVID-19, and approximately 2.4 times more likely to be admitted to an ICU, need mechanical ventilation, or die (16). Such an association was also found for infectious respiratory illnesses. For instance, previous studies have shown that smokers are twice more likely than nonsmokers to have influenza and develop more severe symptoms, while smokers were also noted to have higher mortality rates from the Middle East respiratory syndrome Coronavirus (MERS-CoV) outbreak $(17,18)$. Smoking is additionally detrimental to the respiratory system and its responsiveness to infections, making smokers more prone to severe forms of infectious diseases (16). The severity of COVID19 disease is associated with smoking and leads to the overexpression of the virus-receptor-converting enzyme angiotensin 2 (ACE2), which may increase COVID-19 loading among smokers (19).

Consistent with previous studies, we also found a significant association between increased prevalence of hypertension and COVID-19 mortality. Studies form Wuhan, China, where COVID-19 was emerged, hypertension was found to be associated with disease severity (20-25), such that hypertensive patients were 2 times more likely to demonstrate the severe forms of the disease (20). Zhou et al in a retrospective multicenter cohort study in Wuhan (191 COVID-19 patients) reported that almost half of the patients $(48 \%)$ had at least 1 comorbidity, with hypertension being the most common (30\%), followed by diabetes $(19 \%)$ and coronary heart disease (8\%) (22). In addition, our study showed that the provinces with higher prevalence of diabetes diseases had a higher likelihood of COVID-19-associated deaths, although its association was found to be borderline $(\mathrm{P}$ value $=0.066)$. Chinese studies have shown that the death rates in COVID-19 patients with diabetes increased by $5 \%$, on average $(26,27)$, which is consistent with other studies showing an increased risk of death among patients with diabetes $(24,25,28)$. Both diabetes and hypertension are found to be risk factors for severe clinical outcomes, including death, associated with MERS-CoV, another type of human Coronavirus (29). We could not find a robust significant association between asthma, cancer, and cardiovascular diseases with COVID19 outcomes using province-level data, while such an association was observed in China where the overall case fatality rate was 2.3\% (1023 deaths among 44672 confirmed cases). However, the mortality reached $10.5 \%$ in patients with underlying CVD (30). The mechanism of this association is not yet clear, but it may be due to the increased prevalence of cardiovascular disease in older people, functionally impaired immune system, and increased levels of ACE2 or predisposition to COVID-19 for cardiovascular patients (31). Other studies have also shown increased fatality rate among COVID-19 patients with cancer comorbidity $(25,32,33)$. Data were limited to compare our findings with previous studies in Iran. To our knowledge, only 1 study examined the epidemiological characteristics of COVID-19 cases using individual-level data in a single-center study (11). This study showed that medical comorbidities increased case fatality rates (CFR) of COVID-19, such that CFR among patients without comorbidity $(7.6 \%)$ was lower than that among patients with diabetes $(9.7 \%)$, cardiovascular disease $(10.8 \%)$, chronic respiratory disease $(15.0 \%)$, hypertension $(13.5 \%)$, and chronic kidney diseases (16.7\%). In the same study, patients with any comorbidity were 53\% more likely to die than those without any comorbidity (11).

\section{Limitations}

This study has some limitations. First, our finding from the cross sectional, aggregated data does not infer causality. Second, the "ecological fallacy" (an intrinsic limitation) arising from the ecological studies is indeed possible to occur, such that factors that are correlated with the study outcomes using grouped data may not be correlated with the same diseases using individual-level data (34). However, this might be a serious issue in the current paper as the identified associations/correlations using an ecological design were consistent with and supported by growing body of literature using data from individuals (for example, (11)). Another methodological limitation is that due to the use of grouped aggregated data, we were unable to control the key confounders, such as health service and health professional capacities across the provinces. Due to the small number of analytic units (observation), even their availability could not help us control their role as this may lead to unusable estimates in the regression analyses.

\section{Conclusion}

Our ecological analyses, based on province-level data, show an inequality in COVID-19-associated mortality with health risk factors (ie, obesity and smoking) and underlying medical conditions, particularly hypertension and diabetes. Indeed, further research is warranted to repeat these analyses using individual-level, observational data to support and understand the contribution of these factors to such variations, reduce the burden of the disease, and offer comprehensive approaches to better manage these common conditions during outbreaks from infectious diseases.

\section{Ethical statements}

Ethical approval for this research was obtained from the Research Ethic Committee, Kerman University of Medical Sciences (Code: IR.KMU.REC.1398.735). No written or oral consent form was needed to be obtained from the patients, as this study and its data relied on officially reported administrative data.

\section{Acknowledgments}

We thank the Research Centre for Emerging and Reemerging Infectious Diseases, Pasteur Institute, and the COVID-19 Surveillance Committee in Iran for their help in collecting and provision of the data needed for this ecological study. 


\section{Conflict of Interests}

The authors declare that they have no competing interests.

\section{References}

1. Coronavirus. World Health Organization website https://www.who.int/

health-topics/coronavirus. Published 2020. Accessed March 24, 2020.

2. National Center for Immunization and Respiratory Diseases (NCIRD)

DoVD. Coronavirus disease 2019 (COVID-19) situation summary:

Centers for Disease Control and Prevention website. https://www.cdc.gov/

coronavirus/2019-nCoV/summary.html. Updated March 21, 2020 Accessed

March 24, 2020

3. World Health Organization Novel Coronavirus (2019-nCoV) Situation Report - 12. 2020.

4. Mounesan L, Eybpoosh S, Haghdoost A, Moradi G, Mostafavi E. Is reporting many cases of COVID-19 in Iran due to strength or weakness of Iran's health system? Iran J Microbiol 2020;12(2):73-6.

5. Deng SQ, Peng HJ. Characteristics of and public health responses to the coronavirus disease 2019 outbreak in China. J Clin Med. 2020;9(2):575.

6. Wang T, Du Z, Zhu F, Cao Z, An Y, Gao Y, et al. Comorbidities and multi-organ injuries in the treatment of COVID-19. Lancet. 2020;395(10228):e52.

7. Peeri NC, Shrestha N, Rahman MS, Zaki R, Tan Z, Bibi S, et al. The SARS, MERS and novel coronavirus (COVID-19) epidemics, the newest and biggest global health threats: what lessons have we learned? Int J Epidemiol. 2020.

8. Dietz W, Santos-Burgoa C. Obesity and its Implications for COVID-19 Mortality. Obesity. 2020 Jun;28(6):1005.

9. de la Torre Díez I, Garcia-Zapirain B, Méndez-Zorrilla A, LópezCoronado M. Monitoring and follow-up of chronic heart failure: a literature review of eHealth applications and systems. J Med Syst. 2016;40(7): 179

10. Mehryar S, Sliuzas R, Sharifi A, Reckien D, van Maarseveen M. A structured participatory method to support policy option analysis in a social-ecological system. J Environ Manage. 2017;197:360-72.

11. Nikpouraghdam M, Farahani AJ, Alishiri G, Heydari S, Ebrahimnia M, Samadinia H, et al. Epidemiological Characteristics of Coronavirus Disease 2019 (COVID-19) Patients in IRAN: A single Center Study. J Clin Virol. 2020 Apr 21.

12. Djalalinia S, Modirian M, Sheidaei A, Yoosefi M, Zokaiee H, Damirchilu B, et al. Protocol design for large-scale cross-sectional studies of surveillance of risk factors of non-communicable diseases in Iran: STEPs 2016. Arch Iran Med .2017 Sep 1;20(9).

13. Roshandel G, Ghanbari-Motlagh A, Partovipour E, Salavati F, Hasanpour-Heidari S, Mohammadi G, et al. Cancer incidence in Iran in 2014: results of the Iranian National Population-based Cancer Registry. Cancer Epidemiol. 2019;61:50-8.

14. Ebrahimi H, Pishgar F, Yoosefi M, Moradi S, Rezaei N, Djalalinia S, et al. Insulin pen use and diabetes treatment goals: A study from Iran STEPS 2016 survey. PloS One. 2019;14(8).

15. Centers for disease control and prevention (CDC),National Center for Immunization and Respiratory Diseases (NCIRD), Division of Viral Diseases.Page last reviewed: April 17, 2020.

16. Vardavas CI, Nikitara K. COVID-19 and smoking: A systematic review of the evidence. Tob Induc Dis. 2020;18.

17. Park JE, Jung S, Kim A. MERS transmission and risk factors: a systematic review. BMC Public Health. 2018;18(1):574.

18. Arcavi L, Benowitz NL. Cigarette smoking and infection. Arch Intern Med. 2004;164(20):2206-16.

19. Cai H. Sex difference and smoking predisposition in patients with COVID-19. Lancet Respir Med. 2020;8(4):e20.

20. Alhazzani W, Møller MH, Arabi YM, Loeb M, Gong MN, Fan E, et al. Surviving Sepsis Campaign: guidelines on the management of critically ill adults with Coronavirus Disease 2019 (COVID-19). Intens Care Med. 2020:1-34.

21. Xu Z, Shi L, Wang Y, Zhang J, Huang L, Zhang C, et al. Pathological findings of COVID-19 associated with acute respiratory distress syndrome. Lancet Respir Med. 2020;8(4):420-2.
22. Zhou F, Yu T, Du R, Fan G, Liu Y, Liu Z, et al. Clinical course and risk factors for mortality of adult inpatients with COVID-19 in Wuhan, China: a retrospective cohort study. Lancet. 2020 Mar 11.

23. Yang X, Yu Y, Xu J, Shu H, Liu H, Wu Y, et al. Clinical course and outcomes of critically ill patients with SARS-CoV-2 pneumonia in Wuhan, China: a single-centered, retrospective, observational study. Lancet Respir Med. 2020 Feb 24.

24. Li G, Hu R, Gu X. A close-up on COVID-19 and cardiovascular diseases. Nutrition, Metabolism and Cardiovascular Diseases. 2020.

25. Wang D, Hu B, Hu C, Zhu F, Liu X, Zhang J, et al. Clinical characteristics of 138 hospitalized patients with 2019 novel coronavirus-infected pneumonia in Wuhan, China. JAMA. 2020;323(11):1061-9.

26. Guan WJ, Ni ZY, Hu Y, Liang WH, Ou CQ, He JX, Liu L, Shan H, Lei CL, Hui DS, Du B. Clinical characteristics of coronavirus disease. N Engl J Med. 2019:1708-20.

27. Wu Z, McGoogan JM. Characteristics of and important lessons from the coronavirus disease 2019 (COVID-19) outbreak in China: summary of a report of 72314 cases from the Chinese Center for Disease Control and Prevention. JAMA. 2020 Apr 7;323(13):1239-42.

28. Zhao X, Zhang B, Li P, Ma C, Gu J, Hou P, et al. Incidence, clinical characteristics and prognostic factor of patients with COVID-19: a systematic review and meta-analysis. medRxiv. 2020 Jan 1.

29. Alanazi KH, Abedi GR, Midgley CM, Alkhamis A, Alsaqer T, Almoaddi A, et al. Diabetes Mellitus, Hypertension, and Death among 32 Patients with MERS-CoV Infection, Saudi Arabia. Emerg Infect Dis. 2020;26(1): 166 .

30. Wang W, Xu Y, Gao R, Lu R, Han K, Wu G, et al. Detection of SARS-CoV-2 in different types of clinical specimens. JAMA. 2020 May 12;323(18):1843-4.

31. Clerkin KJ, Fried JA, Raikhelkar J, Sayer G, Griffin JM, Masoumi A, et al. Coronavirus disease 2019 (COVID-19) and cardiovascular disease. Circulation. 2020 May 19;141(20):1648-55.

32. Wang Z, Yang B, Li Q, Wen L, Zhang R. Clinical features of 69 cases with coronavirus disease 2019 in Wuhan, China. Arch Clin Infect Dis. 2020 Mar 16.

33. De Felice F, Polimeni A, Valentini V. The impact of Coronavirus (COVID-19) on head and neck cancer patients' care. Radiother Oncol. 2020;147:84

34. Greenland S, Robins J, Piantadosi S, Cohen B. Ecologic studiesbiases, misconceptions, and counterexamples. Invited commentaries. Authors replies. Am J Epidemiol. 1994;139(8):747-71. 